\title{
T-Wave Alternans and Left Ventricular Wall Thickness in Predicting Arrhythmic Risk in Patients With Hypertrophic Cardiomyopathy
}

\author{
Valentina Otja Puntmann, MD, PhD**; Yee Guan Yap, MD**; \\ William McKenna, MD, PhD*; John Camm, MD
}

\begin{abstract}
Background: Regional heterogeneity of left ventricular (LV) hypertrophy may contribute to arrhythmic vulnerability in patients with hypertrophic cardiomyopathy (HCM). The aim of the present study was to investigate the relationship between LV wall thickness (LVWT) and microvolt T-wave alternans (TWA), a surrogate risk marker of ventricular tachyarrhythmias (VTAs).
\end{abstract}

Methods and Results: A total of 157 consecutive HCM patients underwent 2-D echocardiography and TWAexercise testing, and assessment of arrhythmic burden in a follow up of a median 3.7 years. VTAs were commoner in the non-negative groups (NN-TWA: $n=72$, TWA+ and indeterminate outcome; 29 events, $P<0.02$; TWA+: $n=34$; 14 events, $P=0.01)$, than in the negative TWA group $(n=85,16$ events). TWA+ patients were older $(P<0.04)$ and had greater maximal LVWT and LV mass $(P=0.02$ and $P=0.05$, respectively), whereas NN-TWA linked only with increased LV mass $(P=0.05)$. Regionally, the TWA+ group had greater inferior LVWT $(P<0.05)$. TWA+ outcome positively correlated with maximal LVWT $(r=0.2, P=0.05)$, and basal/equatorial/apical inferior LVWT (BA6: $r=0.2$, $P=0.05$ and $E Q 6: r=0.2 P=0.03, A P 6: r=0.2, P=0.04$ ). Multivariate analysis identified left atrium size, max LVWT and EQ6 with predictive association for TWA+ outcome.

Conclusions: Positive and NN-TWA outcomes are associated with increased LV mass. Moreover, TWA+ is associated with maximal and regional LVWT in HCM patients at risk of arrhythmic events. The present findings support the complementary role of key regional LVWTs in a risk stratification model. (Circ J 2010; 74: 11971204)

Key Words: Hypertrophic cardiomyopathy; Left ventricular wall thickness; Microvolt T-wave alternans; Ventricular tachyarrhythmia

$\mathbf{S}$ riking morphological features with increased left ventricular wall thickness (LVWT) represent the hallmark of the hypertrophic cardiomyopathy (HCM) phenotype ${ }^{1-5}$ yet their relationship with increased risk of ventricular tachyarrhythmias (VTAs) and sudden cardiac death (SCD) in these patients remains poorly characterized. ${ }^{6}$ Previous studies indicated higher risk with greater LVWT, especially with extreme hypertrophy $>30 \mathrm{~mm}$, but patients with mild left ventricular hypertrophy $(\mathrm{LVH})$ are not event free. ${ }^{7-9}$ Thus, morphological measures of LVH appear insufficiently characterized to effectively translate into risk stratification strategies. In addition, marked heterogeneity of LVWT may play a role: recent evidence from our group indicates that in addition to the maximal LVWT, key regional LVWTs may provide complementary information of incremental value to a conventional risk stratification model. ${ }^{10}$

Microvolt T-wave alternans (TWA) emerged as an instrumental non-invasive investigational tool in arrhythmia-prone populations. ${ }^{11-15}$ Measuring the fluctuation in T-wave morphology on the basis of every other (alternate) heart beat is considered to represent spatial or temporal variations in ventricular repolarization, and this has been linked to both inducible and spontaneous VTAs as well as to the mechanisms leading to their initiation. Several lines of evidence support the use of TWA as a reliable predictor of VTAs and arrhythmia event-free survival in at-risk populations, including ischemic, non-ischemic dilated cardiomyopathy. ${ }^{16-19}$ Although a few previous studies investigated the contribution of hypertrophic myocardial phenotype and regional structural variation to the genesis of arrhythmia vulnerability, ${ }^{20,21}$ these relation-

Received December 20, 2009; revised manuscript received February 18, 2010; accepted February 19, 2010; released online May 8, 2010

Time for primary review: 24 days

St George's, University of London, *University College London, Heart Hospital, London, UK

***The first two authors contributed equally to this work.

Mailing address: Valentina Otja Puntmann, MD, PhD, MRCP, St George's, University of London, Cranmer Terrace, London SW17 0RE,

UK. E-mail: vpuntmann@ hotmail.co.uk

ISSN-1346-9843 doi:10.1253/circj.CJ-09-1003

All rights are reserved to the Japanese Circulation Society. For permissions, please e-mail: cj@j-circ.or.jp 

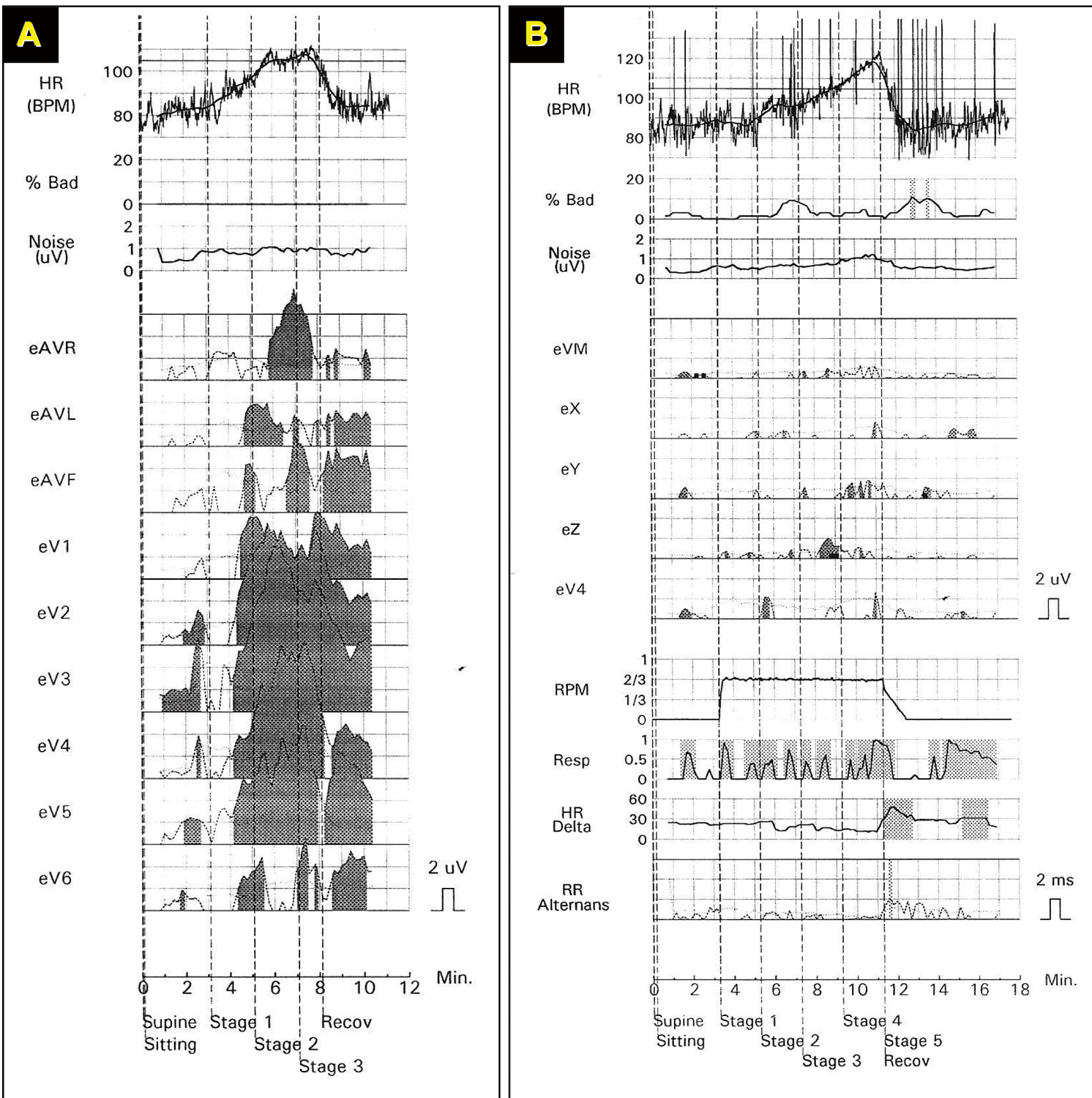

Figure 1. Representative (A) positive and (B) negative microvolt T-wave alternans (TWA) traces on exercise testing using upright bicycle ergometer and a spectral method of analysis designed to allow detection of alternans in the microvolt range of amplitude. Analysis is based on the electrocardiograms (ECGs) recorded from 3 orthogonal $(X, Y, Z)$ and 1 precordial $\left(V_{4}\right)$ leads and the vector magnitude (VM). For an analysis trace, The ECGs were considered valid if the ectopic beats were $<10 \%$ and the noise of the VM was $<1.8 \mu \mathrm{V}$. The magnitude of the TWA was represented as power spectra by calculating the squared magnitude of the fast Fourier transformation of beat-to-beat fluctuations in amplitude of 128 consecutive beats. Traces were regarded as (A) positive with the presence of sustained TWA $>1.9 \mu \mathrm{V}$ for at least $1 \mathrm{~min}$ at the onset heart rate $(\mathrm{HR}) \leq 100$ beats/min. (B) Negative outcome was confirmed when no sustained TWA was present at an HR $\geq 105$ beats/min.

ships remain largely unexplored in the context of HCM..$^{21,22}$ In the present study we investigated the relationship between echocardiographic morphological distribution of LVH in HCM patients and TWA, a surrogate risk marker of VTAs and explored the congruence with our previous findings. ${ }^{10}$

\section{Methods}

\section{Patients}

The study group consisted of 157 consecutive adult patients ( $>16$ years of age) referred to the dedicated HCM clinic at
St George's Hospital, London, UK. The diagnosis of HCM was based on the presence of unexplained LVH > 2SD from the normal range or on the presence of published criteria for the diagnosis of familial disease in the relatives of patients with unequivocal disease. ${ }^{5}$ Informed written consent was obtained from all participants in the study. This study was reviewed and approved by the local ethics committee at St George's Hospital, London, UK.

\section{Clinical Assessment}

Patients underwent clinical assessment including detailed his- 


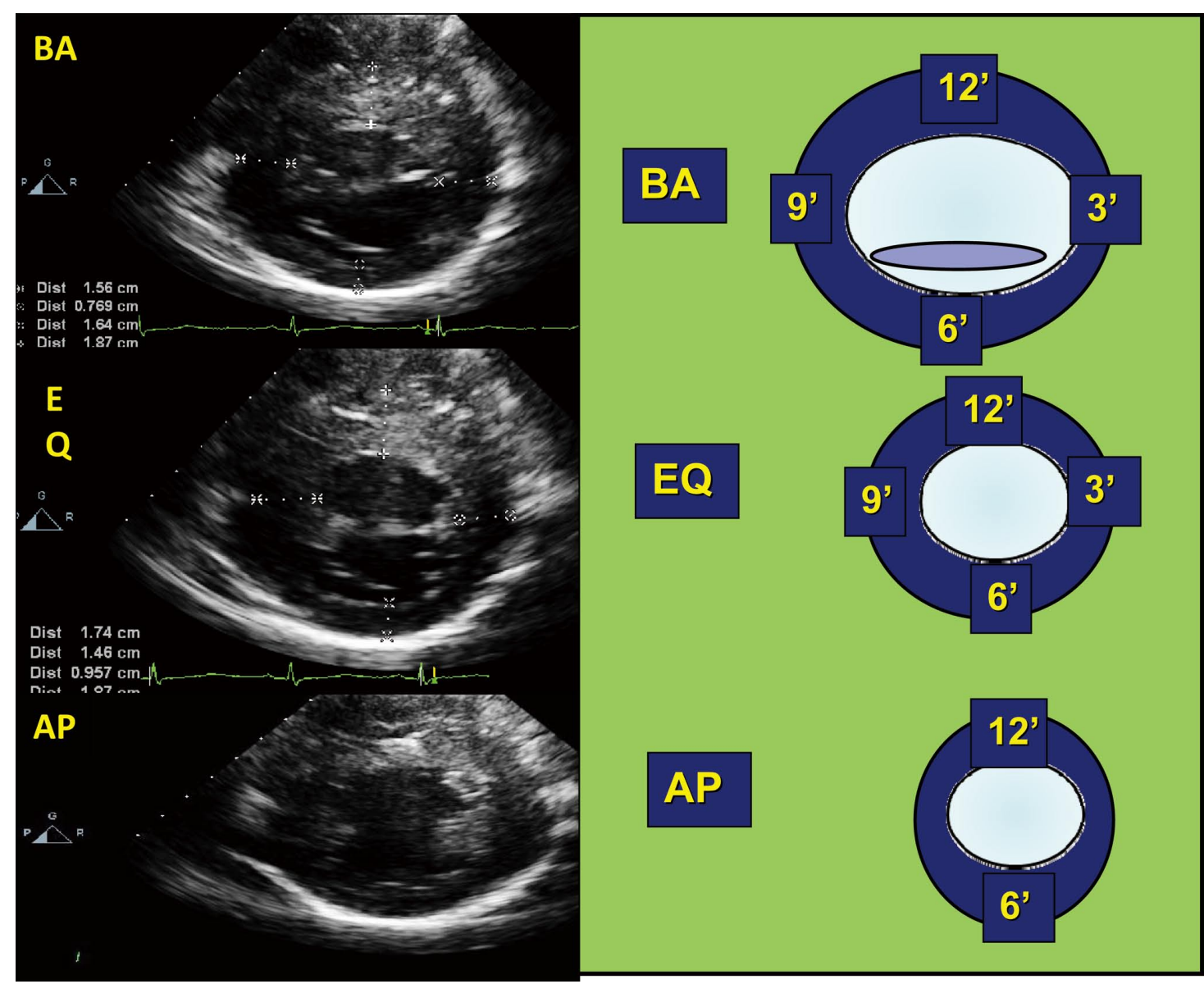

Figure 2. Representative parasternal short-axis slices on 2-D transthoracic echocardiography, used in measurement of hypertrophic load, and schematic representation of corresponding segments. AP, apical slice; BA, basal slice; EQ, equatorial slice.

tory of symptoms (dyspnoe, chest pain, palpitations, syncope) and family history of SCD, HCM, and examination. Dyspnea was classified using the New York Heart Association functional class system. Patients continued their usual medications, including $\beta$-blockers and anti-arrhythmics.

\section{Exercise Test for TWA}

Careful skin preparation, including mild abrasion and highresolution electrodes (High-Res, Cambridge Heart), was used to minimize noise. Electrocardiogram (ECG) leads were placed at the standard 12-lead positions and in an orthogonal $\mathrm{X}, \mathrm{Y}, \mathrm{Z}$ configuration. Stress tests were performed using upright bicycle ergometer (Sensormedics ergometrics 800S, Bitz, Germany) and TWA measurements were made with the CH2000 system (Cambridge Heart), utilizing a spectral analysis designed to allow detection of fluctuating voltage in every other heart beat in the microvolt range of amplitude. The TWA tests were both automatically interpreted by the Alternans Report Classifier (Version D10), but verified by an independent experienced interpreter blinded to the patients' demographics and clinical data in accordance with previously published criteria. ${ }^{23,24}$ For an analysis trace, the ECGs were considered valid if the ventricular ectopic beats were $<10 \%$ and the noise was $<1.8$ microvolt $(\mu \mathrm{V})$. The magnitude of the TWA is represented as power spectra by calculating the squared mag- nitude of the fast Fourier transformation of beat-to-beat fluctuations in amplitude of 128 consecutive beats (Figure 1). Positive TWA outcome is defined as sustained TWA $>1.9 \mathrm{mV}$ at an onset heart rate (HR) $\leq 110$ beats/min, while negative TWA outcome corresponds to the absence of sustained TWA at $\mathrm{HR} \geq 105$ beats/min, for at least $1 \mathrm{~min}$. Any value detected in the range between these two settings was regarded as an indeterminate result. According to the result of TWA testing, comparisons were made between the negative group (TWA-), positive group (TWA+), and non-negative group (NN-TWA: indeterminate group and positive outcome).

\section{Echocardiography}

All patients were imaged in the left lateral decubitus position using an Acuson 128 XP/10 (Mountain View, CA, USA) or GE Vingmed system V (GE Ultrasound Europe). Transthoracic echocardiography was performed using conventional echo windows, imaging planes and standard measurements: indices of global systolic function by biplanar Simpson's ejection fraction (EF), left ventricular (LV) mass (index), and left atrium (LA) size. ${ }^{25,26}$ Maximal and regional LVWTs were obtained in parasternal LV short axis views from $10 \mathrm{LV}$ segments at 3 different levels: basal (BA), equatorial (EQ) and apical level (AP), as depicted in Figure 2. Maximal LVWT was defined as the greatest thickness in any single segment at 


\begin{tabular}{lcccc|}
\hline \multicolumn{1}{l}{ Table 1. Patient Characteristics } & & & & \\
& $\begin{array}{c}\text { TWA- }(\mathbf{n}=\mathbf{8 5}), \\
\mathbf{n}(\%)\end{array}$ & $\begin{array}{c}\text { TWA+ }(\mathbf{n}=\mathbf{3 4}), \\
\mathbf{n}(\%)\end{array}$ & $\begin{array}{c}\text { NN-TWA (n=72), } \\
\mathbf{n}(\%)\end{array}$ & P value \\
Age (years), mean \pm SD & $34.53 \pm 11.8$ & $40.4 \pm 14.8$ & $37.5 \pm 14.5$ & $0.02^{*} / 0.2$ \\
No. men & $47(55.3)$ & $29(85.3)$ & $47(54.7)$ & $0.002^{*} / 0.09$ \\
BSA, mean \pm SD & $1.9 \pm 0.2$ & $1.9 \pm 0.3$ & $1.8 \pm 0.3$ & $0.1 / 0.9$ \\
BMI, mean \pm SD & $24.9 \pm 4.5$ & $25.8 \pm 4.5$ & $25.2 \pm 4.8$ & $0.6 / 0.5$ \\
Clinical symptoms & & & & \\
Dyspnea & $25(29.4)$ & $11(32.4)$ & $23(31.9)$ & $0.4 / 0.09$ \\
FHx of SCD/HCM & $28(32.9)$ & $15(44.9)$ & $27(37.5)$ & $0.3 / 0.3$ \\
Chest pain & $29(25.4)$ & $14(41.2)$ & $22(30)$ & $0.4 / 0.5$ \\
Syncope & $19(22.4)$ & $6(17.6)$ & $13(18.1)$ & $0.6 / 0.3$ \\
\hline
\end{tabular}

${ }^{*} \mathrm{P}<0.05$ considered significant: Student's t-test (TWA+ vs TWA-/NN-TWA vs TWA-).

TWA, T-wave alternans; NN, non-negative; BSA, body surface area; BMI, body mass index; FHx, family history; $\mathrm{SCD} / \mathrm{HCM}$, sudden cardiac death/hypertrophic cardiomyopathy.

\begin{tabular}{|lcccr|}
\hline Table 2. Medication & TWA- $(\mathbf{n = 8 5})$, & $\begin{array}{c}\text { TWA+ }(\mathbf{n}=\mathbf{3 4}), \\
\mathbf{n}(\%)\end{array}$ & $\begin{array}{c}\text { NN-TWA }(\mathbf{n}=\mathbf{7 2}), \\
\mathbf{n}(\%)\end{array}$ & P value \\
None & $\mathbf{n}(\%)$ & $12(35.3)$ & $29(40.7)$ & $0.6 / 0.5$ \\
Ca-antagonists & $37(43.5)$ & $4(11.8)$ & $7(9.7)$ & $0.5 / 0.8$ \\
$\beta$-blockers & $6(7.1)$ & $5(14.7)$ & $11(15.2)$ & $0.05^{\star} / 0.2$ \\
Sotalol & $10(11.7)$ & $3(8.8)$ & $4(5.5)$ & $0.05^{\star} / 0.2$ \\
Amiodarone & $2(2.4)$ & $8(23.5)$ & $14(19.4)$ & $0.05^{\star} / 0.3$ \\
Disopyramydol & $13(15.3)$ & $1(2.9)$ & $2(2.8)$ & $0.2 / 0.3$ \\
\hline
\end{tabular}

${ }^{*} \mathrm{P}<0.05$ is considered significant: Student's t-test (TWA+ vs TWA-/NN-TWA vs TWA-).

Abbreviations see in Table 1.

\begin{tabular}{|c|c|c|c|c|}
\hline Echocardiography & $\begin{array}{c}\text { TWA- }(n=85) \\
\text { mean } \pm \text { SD or } n(\%)\end{array}$ & $\begin{array}{c}\text { TWA+ }(n=34) \\
\text { mean } \pm \text { SD or } n(\%)\end{array}$ & $\begin{array}{c}\text { NN-TWA }(n=72) \\
\text { mean } \pm \text { SD or } n(\%)\end{array}$ & $P$ value \\
\hline LVED & $43.5 \pm 5.8$ & $43.7 \pm 7.1$ & $44.2 \pm 6.5$ & $0.8 / 0.5$ \\
\hline LVES & $25.4 \pm 6.4$ & $25 \pm 6.7$ & $24.9 \pm 6.3$ & $0.7 / 0.7$ \\
\hline Biplanar & $73.6 \pm 10.5$ & $72.8 \pm 9.3$ & $75.6 \pm 11.6$ & $0.5 / 0.6$ \\
\hline FS & $42.2 \pm 9.5$ & $43.6 \pm 8.1$ & $40.7 \pm 11$ & $0.5 / 0.08$ \\
\hline LA size & $39.7 \pm 9.6$ & $45.0 \pm 6.8$ & $42.5 \pm 8.4$ & $0.001^{*} / 0.03^{*}$ \\
\hline LVOT gradient & $23(28.1)$ & $11(32.4)$ & $19(26.4)$ & $0.8 / 0.6$ \\
\hline SAM & $32(37.6)$ & $12(35.3)$ & $25(34.7)$ & $0.9 / 0.3$ \\
\hline \multicolumn{5}{|l|}{ LVH Pattern } \\
\hline ASH & $41(48.2)$ & $18(52.9)$ & $39(54.2)$ & $0.5 / 0.6$ \\
\hline Concentric & $11(12.9)$ & $5(14.7)$ & $12(16.7)$ & $0.4 / 0.6$ \\
\hline Apical & $14(16.5)$ & $4(11.8)$ & 9 (12.5) & $0.2 / 0.3$ \\
\hline Indeterminate & $19(22.4)$ & $8(23.4)$ & $12(16.7)$ & $0.5 / 0.7$ \\
\hline
\end{tabular}

${ }^{*} \mathrm{P}<0.05$ is considered significant: Student's t-test (TWA+ vs TWA-/NN-TWA vs TWA-).

LVED, left-ventricular end-diastolic dimension; LVES, left-ventricular end-systolic dimension; FS, fraction of shortening; LA, left atrium; LVOT, left-ventricular outflow tract; SAM, systolic anterior motion; LVH, left ventricular hypertrophy; ASH, asymmetric septal hypertrophy. Other abbreviations see in Table 1.

the end-diastole. Patterns of hypertrophy, the presence of systolic anterior motion (SAM) and LV outflow tract (LVOT) flow acceleration were defined in accordance with previously published methods. ${ }^{27,28} \mathrm{LV}$ mass was automatically calculated using conventional algorithms by means of inbuilt software and indexed to body surface area (BSA). ${ }^{29}$ TWA testing and ECG were performed within 3 weeks of each other.

\section{Holter Monitoring}

At the study entry and annually thereafter, all patients underwent routine 2-channel (CM1 on channel 1 and $V_{2}$ on channel
2) 48-h ambulatory ECG monitoring (Marquette Electronics, Milwaukee, WI, USA). All arrhythmias, including supraventricular and ventricular origin, were recorded. Ventricular tachycardias (VT) were classified as sustained or non-sustained. Non-sustained VT (NSVT) was defined as $\geq 3$ consecutive ventricular beats at a rate of $\geq 120$ beats/min, lasting for $<30$ s. Patients continued their medication while on Holter monitoring, including $\beta$-blockers and anti-arrhythmic therapy. In addition to annual 48-h Holter recordings, any otherwise documented events on ECG; VT, documented ventricular fibrillation (VF) and $\mathrm{SCD}$, on prospective follow up of a 


\begin{tabular}{|lcccc|}
\hline \multicolumn{5}{l}{ Table 4. Morphological Measures of Global and Regional Hypertrophic Load } \\
Wall thickness & TWA- $(\mathbf{n = 8 5})$ & TWA+ $(\mathbf{n}=\mathbf{3 4})$ & NN-TWA $(\mathbf{n = 7 2})$ & P value \\
LVmass & $210.5 \pm 78.6$ & $252.3 \pm 92.4$ & $238.5 \pm 10.5$ & $0.02^{*} / 0.05^{*}$ \\
LVmass index & $117.7 \pm 53.7$ & $133.7 \pm 58.1$ & $130.6 \pm 57.8$ & $0.1 / 0.2$ \\
Max LVWT & $18.2 \pm 5.8$ & $21.6 \pm 6.0$ & $19.8 \pm 6.7$ & $0.05^{*} / 0.1$ \\
BA LVWT12 & $14.6 \pm 4.6$ & $16.2 \pm 5.3$ & $14.6 \pm 4.6$ & $0.09 / 0.1$ \\
BA LVWT9 & $13.6 \pm 4.7$ & $14.1 \pm 3.7$ & $16.9 \pm 7.9$ & $0.6 / 0.1$ \\
BA LVWT6 & $10.8 \pm 2.7$ & $12 \pm 2.9$ & $12.8 \pm 7.2$ & $0.05^{*} / 0.3$ \\
BA LVWT3 & $13.2 \pm 3.2$ & $14.7 \pm 3.8$ & $13.9 \pm 3.9$ & $0.1 / 0.3$ \\
EQ LVWT12 & $16.6 \pm 5.9$ & $18.6 \pm 6$ & $19.9 \pm 8.8$ & $0.1 / 0.2$ \\
EQ LVWT9 & $15.5 \pm 5.3$ & $16 \pm 5.2$ & $20.1 \pm 9.8$ & $0.7 / 0.1$ \\
EQ LVWT6 & $11.4 \pm 2.9$ & $12.7 \pm 2.8$ & $11.7 \pm 3.3$ & $0.03^{*} / 0.7$ \\
EQ LVWT3 & $13.6 \pm 3$ & $14.1 \pm 3.5$ & $18.5 \pm 7.5$ & $0.6 / 0.3$ \\
AP LVWT12 & $15.2 \pm 6.3$ & $17.9 \pm 6.7$ & $21.1 \pm 5.8$ & $0.09 / 0.1$ \\
AP LVWT6 & $12.7 \pm 4.5$ & $15 \pm 4.7$ & $17.8 \pm 8.3$ & $0.03^{*} / 0.2$ \\
\hline
\end{tabular}

${ }^{*} \mathrm{P}<0.05$ is considered significant: Student's t-test (TWA+ vs TWA-/NN-TWA vs TWA-).

LVWT, left ventricular wall thickness; BA, basal slice; LVWT-number, o'clock position in parasternal short-axis view; $E Q$, equatorial slice; $A P$, apical slice. Other abbreviations see in Table 1.

\begin{tabular}{|c|c|c|c|c|}
\hline Arrhythmic capture & $\begin{array}{c}\text { TWA- }(\mathrm{n}=85), \\
\mathrm{n}(\%)\end{array}$ & $\begin{array}{c}\text { TWA+ }(n=34) \\
n(\%)\end{array}$ & $\begin{array}{c}\text { NN-TWA }(\mathrm{n}=72), \\
\mathrm{n}(\%)\end{array}$ & $P$ value \\
\hline Total VTAs & $16(18.8)$ & $14(41.2)$ & $29(40.1)$ & $0.01^{*} / 0.02^{*}$ \\
\hline Hard VTAs & $7(8)$ & $1(3)$ & $8(11)$ & $0.4 / 0.9$ \\
\hline VF (aborted) & $3(3.5)$ & $0(0)$ & $5(7)$ & $0.08 / 0.3$ \\
\hline SCD & $4(4.9)$ & $1(3)$ & $3(4.2)$ & $0.6 / 0.9$ \\
\hline NSVT & $9(10.6)$ & $13(38.2)$ & $21(29.2)$ & $0.02^{*} / 0.1$ \\
\hline VES grade $>3$ & $22(25.3)$ & $12(33.4)$ & $25(34.7)$ & $0.1 / 0.1$ \\
\hline VES (total $n$ ), mean \pm SD & $369.7 \pm 965.5$ & $507.7 \pm 2,983$ & $539 \pm 2,878$ & $0.7 / 0.9$ \\
\hline Abnormal BP response & $19(22.4)$ & $8(23.5)$ & $20(30)$ & $0.1 / 0.4$ \\
\hline
\end{tabular}

${ }^{*} \mathrm{P}<0.05$ is considered significant: Student's t-test (TWA+ vs TWA-/NN-TWA vs TWA-).

VTA, ventricular tachyarrhythmia; total VTA, composite of ventricular fibrillation (VF), non-sustained ventricular tachycardia (NSVT) and sudden cardiac death (SCD); Hard VTA, VF and SCD only; VES, ventricular ectopic beats; $\mathrm{BP}$, blood pressure. Other abbreviations see in Table 1.

median 3.7 years (range $0-143$ months) were included into a total VTAs endpoint. SCD, VF and sustained VTs counted as the conventional hard VTAs endpoint. ${ }^{23,24,30}$

\section{Statistical Analysis}

For comparison of 2 normally distributed variables, we used Student's t-test. The chi-squared test was utilized to compare non-continuous variables expressed as proportions, with the Fisher exact test as appropriate for the type of data. Analysis of relationships between TWA outcome and various variables was made using correlations (Pearson's correlation coefficient for normally distributed variables and Spearman's correlation coefficient for non-parametric data), binary logistic regression analysis (positive, non-negative vs negative TWA outcome) with stepwise selection of clinical variables to reduce the model to only statistically significant parameters. Data are expressed as mean \pm SD. All tests were two-tailed; $\mathrm{P}<0.05$ was considered significant. SPSS statistical software (SPSS, Chicago, IL, USA, version 15.0) was used for the statistical analysis.

\section{Results}

Clinical Characteristics

Clinical characteristics of the study population are presented in Table 1 . In the TWA+ group there were proportionally more male and older patients who used more amiodarone and $\beta$-blockers (Table 2).

\section{Transthoracic Echocardiography}

There were no significant differences among the conventional echocardiographic measures (Table 3), including intra-cavity dimensions, presence of SAM or LVOT gradient, or pattern of LVH distribution among the groups. The LA size was significantly larger in both non-negative TWA groups. All groups had supernormal global systolic function and hyperdynamic radial systolic function, expressed as fraction of shortening.

\section{Maximal and Regional Wall Thickness}

Despite the balanced distribution of conventional patterns of hypertrophy among all groups, ${ }^{3}$ the non-negative groups had significantly raised LV mass. Moreover, TWA+ patients had significantly greater maximal LVWT. Regionally, the TWA+ group had distinctively larger inferior LVWT in all 3 parasternal short axis levels (Table 4).

\section{Cumulative Arrhythmic Capture}

VTAs were significantly more common in both TWA+ and non-negative TWA groups (Table 5). Notably, there was 


\begin{tabular}{|c|c|c|c|c|}
\hline & HR & Wald & $P$ value & $95 \% \mathrm{Cl}$ \\
\hline Age & 1 & 0.3 & 0.6 & $0.9-1.0$ \\
\hline Gender (male) & 6.1 & 8.9 & $0.003^{*}$ & $1.9-9$ \\
\hline $\mathrm{FHx}$ of SCD/HCM & 1.4 & 0.9 & 0.4 & $0.6-3.2$ \\
\hline Chest pain & 0.9 & 0.05 & 0.9 & $0.8-1.1$ \\
\hline Dyspnea (NYHA class >I) & 1 & 0.6 & 0.7 & $0.8-1.3$ \\
\hline Syncope & 0.9 & 2 & 0.4 & $0.7-1.1$ \\
\hline Palpitations & 0.8 & 0.3 & 0.6 & $0.3-2.0$ \\
\hline Max LVWT & 1.1 & 2.1 & 0.08 & $0.9-5.1$ \\
\hline LA size & 1.0 & 0.7 & 0.6 & $0.9-3.1$ \\
\hline LVED & 0.9 & 0.03 & 0.8 & $0.7-1.2$ \\
\hline LVES & 0.9 & 0.1 & 0.9 & $0.7-1.3$ \\
\hline FS & 1.1 & 0.1 & 0.9 & $0.8-1.3$ \\
\hline LVH pattern & 0.9 & 0.2 & 0.9 & $0.8-1.8$ \\
\hline SAM & 2.8 & 0.1 & 0.9 & $0.6-9.2$ \\
\hline Mid-cavity gradient & 0.9 & 0.2 & 0.7 & $0.2-2.5$ \\
\hline LVOT Vmax & 0.9 & 0.1 & 0.9 & $0.4-1.5$ \\
\hline BA LVWT12 & 0.3 & 0.9 & 0.8 & $0.1-1.1$ \\
\hline BA LVWT 9 & 0.9 & 0.3 & 0.9 & $0.8-1.2$ \\
\hline BA LVWT 6 & 1.1 & 2.6 & 0.07 & $0.8-4.2$ \\
\hline BA LVWT 3 & 1.2 & 0.3 & 0.5 & $0.8-1.5$ \\
\hline EQ LVWT 12 & 0.9 & 0.1 & 0.9 & $0.7-1.3$ \\
\hline EQ LVWT 9 & 1 & 0.1 & 0.5 & $0.8-1.3$ \\
\hline EQ LVWT 6 & 1.2 & 4.1 & $0.01^{*}$ & $1.0-1.3$ \\
\hline EQ LVWT 3 & 1 & 0.6 & 0.9 & $0.7-1.2$ \\
\hline AP LVWT 12 & 1.8 & 0.1 & 0.8 & $0.8-7.9$ \\
\hline AP LVWT 6 & 1.2 & 3.9 & $0.02^{*}$ & $0.9-1.4$ \\
\hline
\end{tabular}

${ }^{*} \mathrm{P}<0.05$ is considered significant.

$\mathrm{HR}$, hazard ratio; $\mathrm{Cl}$, confidence interval; NYHA, New York Heart Association; Vmax, Doppler maximal flow velocity. Other abbreviations see in Tables 1,3,4.

significantly higher frequency of NSVT in TWA+, whereas the NN-TWA group had a non-significant representation of documented VFs.

\section{Analysis of the Relationships}

TWA+ outcome positively correlated with the total VTAs score $(r=-0.2, P=0.01)$, greater LA size $(r=0.3, P=0.009)$, maximal LVWT $(r=0.2, P=0.05)$ and with inferior LVWT at the 3 short axis levels (BA6: $r=0.2, P=0.05$; EQ6: $r=0.2$, $\mathrm{P}=0.03$; AP6: $\mathrm{r}=0.2, \mathrm{P}=0.04)$. Although univariate analysis identified male gender, EQ6 and EQ3 as the significant sole predictors of TWA+ outcome (Table 6), we were able to construct 2 significant models with predictive association for TWA+ using LA size, max LVWT and EQ6 (Table 7). No such links were observed for NN-TWA outcome. While TWA+ outcome closely correlated with maximal LVWT and regional heterogeneity of LVWT, especially in the inferior wall, we failed to find a relationship between TWA outcomes and hard VTAs endpoint.

\section{Discussion}

There are several key findings to this study. First, we demonstrate a significant relationship between increase in LV mass and the non-negative TWA groups, in line with previous findings for arrhythmic association with increased hypertrophic load. ${ }^{5-10}$ Next, TWA+ outcome links with not only maximal, but also regional LVWT in patients with HCM, corroborating the previous finding that regional information may improve characterization of the arrhythmic risk, associated with increased LVWT. ${ }^{10}$ Last, predictive association of LVWT of the key segments with TWA+ outcome provide important new evidence on arrhythmia vulnerability in HCM with complementary information over and above a traditional risk stratification model in this susceptible population.

\section{Mechanistic Link Between TWA and LVWT in HCM}

To the best of our knowledge this is the first study that provides evidence that $\mathrm{LV}$ regional morphological differences in patients with HCM are related to the TWA outcome and potentially reflect the arrhythmic susceptibility from regional heterogeneity. Moreover, these results expand on the concept that microvolt TWA has the ability to detect significant regional/spatial heterogeneity in the progression of repolarization, possibly reflecting the underlying structural irregularities. ${ }^{13,14}$ Few previous studies investigated the contribution of prominent myocardial phenotype and regional morphological variation to the genesis of arrhythmia susceptibility in relation to the TWA phenomenon in hypertrophic populations. In hypertensive LVH, the action potential duration or rate-dependent QT interval were shown to be inappropriately long, causing action potential alternans in hypertrophied myocytes and TWA in these patients, suggesting that increased LVWT does interact with the repolarization process. ${ }^{30-33}$ Investigation of regional TWA heterogeneity in patients with hypertensive systolic dysfunction provided additional correlates between TWA and distinct structural entities such as eccentric LVH, regional myocardial scar underscoring sys-

\begin{tabular}{|c|c|c|c|c|c|c|c|}
\hline \multirow{3}{*}{$\begin{array}{l}\text { TWA+ vs TWA- } \\
\text { Model } 1(\mathrm{P}=0.001)\end{array}$} & \multirow{2}{*}{ B } & \multirow{2}{*}{ SE } & \multirow{2}{*}{ d.f. } & \multirow{2}{*}{$P$ value } & \multirow{2}{*}{ OR } & \multicolumn{2}{|c|}{$95 \% \mathrm{Cl}$ for OR } \\
\hline & & & & & & Lower & Upper \\
\hline & \multicolumn{3}{|c|}{ Cox\&Snell $R^{2}=19 \%$} & \multicolumn{2}{|c|}{ Nagelkerke $R^{2}=26 \%$} & & \\
\hline LA size & 0.1 & 0.04 & & 0.9 & 1 & 0.9 & 1.1 \\
\hline Maximal LVWT & 0.007 & 0.05 & 1 & 0.008 & 1.1 & 0.8 & 1.1 \\
\hline BA LVWT6 & 0.2 & 0.1 & 1 & 0.1 & 0.8 & 0.7 & 0.9 \\
\hline EQ LVWT6 & 0.1 & 0.1 & 1 & 0.05 & 1.1 & 0.9 & 1.2 \\
\hline AP LVWT6 & 0.1 & 0.06 & 1 & 0.05 & 1.1 & 0.9 & 1.1 \\
\hline Model $2(P=0.001)$ & \multicolumn{3}{|c|}{ Cox\&Snell $R^{2}=18 \%$} & \multicolumn{3}{|c|}{ Nagelkerke $\mathrm{R}^{2}=25 \%$} & \\
\hline LA size & 0.08 & 0.03 & 1 & 0.007 & 1.1 & 1.02 & 1.2 \\
\hline EQ LVWT6 & 0.1 & 0.05 & 1 & 0.01 & 1.1 & 1.0 & 1.2 \\
\hline
\end{tabular}

OR, odds ratio. Other abbreviations see in Tables 1,3,4,6. 
tolic dysfunction, and the tendency for inducible ventricular arrhythmias. ${ }^{34}$ These investigators showed that the link between positive regional TWA and underlying myocardial scar held a predictive association for the regional distribution. Another study that paralleled hypertensive LVH with HCM of a similar grade showed differential TWA profiles with higher microvolt magnitudes in HCM patients, which were especially diverse in the presence of marked underlying histopathology (myocardial disarray and/or fibrosis). ${ }^{31}$ Thus, previous evidence suggests that TWA may have the capacity to reflect arrhythmic susceptibility and regional heterogeneity of thickness and histopathology. ${ }^{31}$

\section{TWA and Regional LVH in HCM}

Recent evidence from our group suggests that regional contribution of hypertrophy in HCM patients adds to arrhythmia susceptibility in this population. ${ }^{10} \mathrm{HCM}$ patients with arrhythmic events display remarkably greater overall maximal LVWT, and patients with raised maximal LVWT had significantly increased risk of VTAs. Yet, maximal LVWT, that is, the greatest LVWT anywhere in the LV, provided a meaningful contribution only in conjunction with traditional risk factors, such as family history of SCD/HCM or adverse blood pressure response to exercise. Regional distribution of LVWT appears to play a more relevant role and regional measures, BA12 and EQ6 LVWTs were in fact identified as independent predictors of arrhythmic risk, over and above the conventional risk stratification model. The findings of the present study whereby TWA+ outcome, a surrogate marker of arrhythmia vulnerability, links with increased inferior LVWT further corroborate this evidence. Given that TWA is caused by underlying regional inhomogeneities of ventricular repolarization, which predispose patients to VTAs, ${ }^{31}$ it is conceivable that regional determination may have a more relevant role in susceptibility to the VTA over the prominent hypertrophic phenotype. Moreover, several lines of evidence indicate the presence of predilection sites in HCM with prominent regional fibrosis in the form of confluent multifocal, subendocardial patches particularly in the inter-ventricular junctions. ${ }^{35}$ Increased LVWT in the inferior wall may well reflect the patches of regional fibrosis. Evidence suggests that increased fibrotic load affords for the greater susceptibility of arrhythmic events in the HCM population. ${ }^{36}$ Whether increased inferior LVWT reflects amassed regional fibrosis and its direct involvement in arrhythmia vulnerability and/or TWA outcomes remains to be ascertained in future studies.

In line with previous studies in arrhythmia-prone populations, positive TWA outcome failed to segregate the patients' hard VTAs, such as VF and SCD. Positive TWA outcome, however, linked significantly with the total VTA endpoint including the NSVTs, an arrhythmic subset with some established relevance to the risk of SCD in the HCM population. ${ }^{36,37}$ Despite extensive testing in laboratory and clinical studies in arrhythmia-prone populations, it appears that TWA testing is capable of detecting increased cardiac electrical instability and risk for arrhythmia, but lacks coherence in indentification of what is commonly considered a malignant arrhythmic subset. Evidence suggests that the potential usefulness of this methodology in risk stratification lies in its high negative predictive value (NPV). Metanalysis by Gehi et al reported a high NPV of $97.2 \%$ with a respectable confidence interval of 96.5-97.9, while positive PV (PPV) varied significantly depending on the population studied. ${ }^{38}$ While the present study used TWA testing in an exploration of gen- eral arrhythmic susceptibility and did not primarily attempt to test the role of arrhythmic subsets in relation to SCD in $\mathrm{HCM}$, the use of this methodology as a surrogate marker of arrhythmic vulnerability is likely justified. Future studies are needed to improve the understanding and translation between various arrhythmic subsets and TWA outcomes, if any.

\section{Clinical Implication and Future Studies}

The role of non-invasive imaging in identification of highrisk HCM patients remains an evolving field of crucial clinical utility. This study provides important new evidence that regional hypertrophy in HCM may represent an electrophysiological substrate for pro-arrhythmia in a heterogeneous distribution. Thus, routine non-invasive morphological examination of LV in HCM may benefit from characterization of regional morphology, such as regional LVWT of the inferior wall. In combination with TWA testing, this may identify the HCM patients with positive TWA and regional hypertrophy at the highest risk of arrhythmic events. Further studies are required to fully translate the potential of this strategy into identification of HCM patients at risk of SCD. Similarly, regional electrophysiological studies combined with regional tissue characterization imaging or histology to further decipher the observed patterns in the underlying arrhythmogenic substrate are needed.

\section{Study Limitations}

A number of limitations apply to the present study. First, previous studies linking the regional magnitude of TWA voltage, the extent of hypertrophy and regional heterogeneity in the presence of myocardial scar, have been predictive only in the magnitude range above the positive threshold. ${ }^{36}$ This supports the validity of established TWA definitions ${ }^{22,23}$ and, hence, our decision to explore the relevant TWA information non-regionally, within the dichotomous divide between the non-negative and negative outcome. Next, annual Holter monitoring may have captured only a limited amount of VTAs in the present HCM population. Despite the rigorous protocol and consistent application in this study population, our arrhythmic capture may have provided only a subset of the total arrhythmic burden.

\section{Conclusions}

Positive and NN-TWA outcomes, surrogates of arrhythmic vulnerability, are associated with increased LV mass. Moreover, TWA+ has associations with maximal and regional LVWT in HCM patients at risk of arrhythmic events. The present findings support the complementary role of key regional LVWT in a risk stratification model.

\section{Acknowledgments}

Dr Y. G. Yap was a British Heart Foundation Research Fellow in Cardiology. Professor A. J. Camm is British Heart Foundation Professor of Clinical Cardiology.

\section{Disclosures}

Authors report no conflicts of interest.

\section{References}

1. Spirito P, Seidman CE, McKenna WJ, Maron BJ. The management of hypertrophic cardiomyopathy. N Engl J Med 1997; 336: $775-$ 785 .

2. Wigle ED, Sasson Z, Henderson MA, Ruddy TD, Fulop J, Rakowski H, et al. Hypertrophic cardiomyopathy: The importance 
of the site and the extent of hypertrophy: A review. Prog Cardiovasc Dis 1985; 28: 1-83.

3. Maron BJ, Epstein SE. Hypertrophic cardiomyopathy: Recent observations regarding the specificity of three hallmarks of the disease: Asymmetric septal hypertrophy, septal disorganization and systolic anterior motion of the anterior mitral leaflet. Am J Cardiol 1980; 45: $141-154$.

4. Richardson P, McKenna W, Bristow M, Maisch B, Mautner B, O'Connell J, et al. Report of the 1995 World Health Organization/ International Society and Federation of Cardiology Task Force on the definition and classification of cardiomyopathies. Circulation 1996; 93: $841-842$.

5. Elliott P, Andersson B, Arbustini E, Bilinska Z, Cecchi F, Charron $\mathrm{P}$, et al. Classification of the cardiomyopathies: A position statement from the European Society Of Cardiology Working Group on Myocardial and Pericardial Diseases. Eur Heart J 2008; 29: $270-$ 276.

6. Kunavarapu C, Bloomfield DM. Role of non-invasive studies in risk stratification for sudden cardiac death. Clin Cardiol 2004; 27: $192-197$.

7. Spirito P, Bellone P, Harris KM, Bernabo P, Bruzzi P, Maron BJ. Magnitude of left ventricular hypertrophy and risk of sudden death in hypertrophic cardiomyopathy. N Engl J Med 2000; 342: 1778 1785.

8. Elliott PM, Gimeno Blanes JR, Mahon NG, Poloniecki JD, McKenna WJ. Relation between severity of left-ventricular hypertrophy and prognosis in patients with hypertrophic cardiomyopathy. Lancet 2001; 357: 420-424.

9. Olivotto I, Maron MS, Autore C, Lesser JR, Rega L, Casolo G, et al. Assessment and significance of left ventricular mass by cardiovascular magnetic resonance in hypertrophic cardiomyopathy. $J \mathrm{Am}$ Coll Cardiol 2008; 52: 559-566.

10. Puntmann VO, Yap YG, McKenna W, Camm AJ. Significance of maximal and regional $\mathrm{LV}$ wall thickness in association with arrhythmic events in patients with hypertrophic cardiomyopathy. Circ $J$ 2010; 74: 531-537.

11. Pham Q, Quan KJ, Rosenbaum DS. T-wave alternans: Marker, mechanism, and methodology for predicting sudden cardiac death. J Electrocardiol 2003; 36(Suppl): 75-81.

12. Pastore JM, Girouard SD, Laurita KR, Akar FG, Rosenbaum DS. Mechanism linking T-wave alternans to the genesis of cardiac fibrillation. Circulation 1999; 99: 1385-1394.

13. Chinushi M, Restivo M, Caref EB, El-Sherif N. Electrophysiological basis of arrhythmogenicity of QT/T alternans in the long-QT syndrome: Tridimensional analysis of the kinetics of cardiac repolarization. Circ Res 1998; 83: 614-628.

14. Narayan SM. T-wave alternans and the susceptibility to ventricular arrhythmias. J Am Coll Cardiol 2006; 47: 269-281.

15. Bloomfield DM, Steinman RC, Namerow PB, Parides M, Davidenko J, Kaufman ES, et al. Microvolt T-wave alternans distinguishes between patients likely and patients not likely to benefit from implanted cardiac defibrillator therapy: A solution to the Multicenter Automatic Defibrillator Implantation Trial (MADIT) II conundrum. Circulation 2004; 110: 1885-1889.

16. Bloomfield DM, Bigger JT, Steinman RC, Namerow PB, Parides MK, Curtis AB, et al. Microvolt T-wave alternans and the risk of death or sustained ventricular arrhythmias in patients with left ventricular dysfunction. J Am Coll Cardiol 2006; 47: 456-463.

17. Maeda S, Nishizaki M, Yamawake N, Ashikaga T, Shimada H, Asano M, et al. Ambulatory ECG-based T-wave alternans and heart rate turbulence predict high risk of arrhythmic events in patients with old myocardial infarction. Circ J 2009; 73: 2223-2228.

18. Kim JW, Pak HN, Park JH, Nam GB, Kim SK, Lee HS, et al. Defibillator electrogram $\mathrm{T}$ wave alternans as a predictor of spontaneous ventricular tachyarrhythmias in defibrillator recipients. Circ J 2009; 73: $55-62$.

19. Klingenheben T, Ptaszynski P, Hohnloser S. Quantitative assessment of microvolt $\mathrm{T}$-wave alternans in patients with congestive heart failure. J Cardiovasc Electrophysiol 2005; 16: 620-624.

20. Pastore JM, Rosenbaum DS. Role of structural barriers in the mechanism of alternans-induced reentry. Circ Res 2000; 87: 1157-1163.
21. Momiyama Y, Hartikainen J, Nagayoshi H, Albrecht P, Kautzner $\mathrm{J}$, Saumarez RC, et al. Exercise-induced T-wave alternans as a marker of high risk in patients with hypertrophic cardiomyopathy. Circ J 1997; 61: 650-656.

22. Kuroda N, Ohnishi Y, Yoshida A, Kimura A, Yokoyama M. Clinical significance of T-wave alternans in hypertrophic cardiomyopathy. Circ J 2002; 66: 457-462.

23. Bloomfield DM, Hohnloser SH, Cohen RJ. Interpretation and classification of microvolt T-wave alternans tests. J Cardiovasc Electrophysiol 2002; 13: 502-512.

24. Richter S, Duray G, Hohnloser SH. How to analyze T-wave alternans. Heart Rhythm 2005; 2: 1268-1271.

25. Cheitlin MD, Armstrong WF, Aurigemma GP, Beller GA, Bierman FZ, Davis JL, et al. A report of the American College of Cardiology/American Heart Association Task Force on Practice Guidelines (ACC/AHA/ASE Committee Update the 1997 Guidelines on the Clinical Application of Echocardiography). Circulation 2003; 108: $1146-1162$.

26. Gardin JM, Adams DB, Douglas PS, Feigenbaum H, Forst DH, Fraser AG, et al; American Society of Echocardiography. Recommendations for a standardized report for adult transthoracic echocardiography: A report from the American Society of Echocardiography's Nomenclature and Standards Committee and Task Force for a Standardized Echocardiography Report. J Am Soc Echocardiogr 2002; 15: 275-290.

27. Shapiro LM, McKenna WJ. Distribution of left ventricular hypertrophy in hypertrophic cardiomyopathy: A two-dimensional echocardiographic study. J Am Coll Cardiol 1983; 2: 437-444.

28. Elliott P, Gimeno J, Tomé M, McKenna W. Left ventricular outflow tract obstruction and sudden death in hypertrophic cardiomyopathy. Eur Heart J 2006; 27: 3073.

29. Lang RM, Bierig M, Devereux RB, Flachskampf FA, Foster E, Pellikka PA, et al; American Society of Echocardiography's Nomenclature and Standards Committee; Task Force on Chamber Quantification; American College of Cardiology Echocardiography Committee; American Heart Association; European Association of Echocardiography, European Society of Cardiology. Recommendations for chamber quantification. Eur J Echocardiogr 2006; 2: $79-108$.

30. Rosenbaum D. T-wave alternans in the sudden cardiac death in heart failure trial population: Signal or noise? Circulation 2008; 118: $2015-2018$.

31. Kon-No Y, Watanabe J, Koseki Y, Koyama J, Yamada A, Toda S, et al. Microvolt $\mathrm{T}$ wave alternans in human cardiac hypertrophy: Electrical instability and abnormal myocardial arrangement. J Cardiovasc Electrophysiol 2001; 12: 759-763.

32. Hennersdorf MG, Niebch V, Perings C, Strauer BE. T wave alternans and ventricular arrhythmias in arterial hypertension. Hypertension 2001; 37: 199-203.

33. Keung ECH, Aronson RS. Non-uniform electrophysiologic properties and electrotonic interaction in hypertrophied rat myocardium. Circ Res 1981; 49: 150-158.

34. Narayan SM, Smith JM, Lindsay BD, Cain ME, Dávila-Román VG. Relation of T-wave alternans to regional left ventricular dysfunction and eccentric hypertrophy secondary to coronary heart disease. Am J Cardiol 2006; 97: 775-780.

35. Moon JC, McKenna WJ, McCrohon JA, Elliott PM, Smith GC, Pennell DJ. Toward clinical risk assessment in hypertrophic cardiomyopathy with gadolinium cardiovascular magnetic resonance. J Am Coll Cardiol 2003; 41: 1561-1567.

36. Kwon DH, Smedira NG, Rodriguez ER, Tan C, Setser R, Thamilarasan M, et al. Cardiac magnetic resonance detection of myocardial scarring in hypertrophic cardiomyopathy: Correlation with histopathology and prevalence of ventricular tachycardia. $J \mathrm{Am}$ Coll Cardiol 2009; 54: 242-249.

37. McKenna WJ, England D, Doi YL, Deanfield JE, Oakley C, Goodwin JF. Arrhythmia in hypertrophic cardiomyopathy. I: Influence on prognosis. Br Heart J 1981; 46: $168-172$.

38. Gehi AK, Stein RH, Metz LD, Gomes JA. Microvolt T-wave alternans for the risk stratification of ventricular tachyarrhythmic events: A meta-analysis. J Am Coll Cardiol 2005; 46: 75-82. 